\title{
DETERMINATION OF ORIENTATION AND ALLOYING EFFECTS ON CREEP RESPONSE AND DEFORMATION MECHANISMS IN SINGLE CRYSTALS OF Ni-BASE DISK SUPERALLOYS
}

\author{
T.M. Smith ${ }^{1,2}$, L.V. Duchao ${ }^{2}$, T. Hanlon ${ }^{3}$, A. Wessman ${ }^{4}$, Y. Wang ${ }^{2}$, M.J. Mills ${ }^{1,2}$ \\ ${ }^{1}$ Center for Electron Microscopy and Analysis, The Ohio State University, Columbus, OH 43212, USA \\ ${ }^{2}$ Department of Materials Science and Engineering, The Ohio State University, Columbus, OH 43210, USA \\ ${ }^{3}$ GE Global Research, Niskayuna, NY 12309, USA \\ ${ }^{4}$ GE Aviation, Cincinnati OH 45215, USA
}

Keywords: Ni-based Superalloy, Compression Creep, STEM, Deformation Mechanisms, Dislocation Activity Diagram

\begin{abstract}
In this study, the effects of varying crystal orientation and composition on active deformation modes are explored for two different Ni-base disk alloys. Understanding these effects will allow for improved predictive modeling and consequently faster advancements in Ni-base alloy development. In order to investigate these effects, compression creep tests were conducted on [001] and [110] oriented single crystal specimens of the disk alloys ME3 and ME501, at different stress/temperature regimes. At $760{ }^{\circ} \mathrm{C}$ and below, a prominent creep anisotropy exists between the two orientations, with the [110] oriented samples exhibiting superior creep strength. At $815^{\circ} \mathrm{C}$, the creep anisotropy disappeared between the two orientations. Through bright field scanning transmission electron microscopy, it was determined that the existence of creep anisotropy is a result of differences in deformation modes between the different orientations and alloy compositions. Results of phase field modeling in which the interaction of dislocations with realistic precipitate structures is also conducted to further advance predictive creep deformation models.
\end{abstract}

\section{Introduction}

Ni-base superalloys have long been the standard for use in the hot section of turbine engines. However, as the operating temperatures in next generation engines are increased, in order to improve engine efficiency and reduce carbon emissions, new deformation modes may become dominant and strongly affect the creep behavior. Improved understanding of these mechanisms will help progress the understanding of deformation in this regime, and drive improvements in the high temperature properties of new generation Ni-base superalloys.

\section{Creep Deformation at intermediate temperatures}

Detailed studies of the mechanism changes in these polycrystalline Ni-base alloys in different temperature and stress regimes have proven to be a daunting endeavor, especially at intermediate temperatures between $650-800{ }^{\circ} \mathrm{C}$. Presently little is understood about these deformation mechanisms and the parameters that affect their operation. It is widely agreed that below the intermediate temperature range athermal shearing by full $1 / 2<110>$ dislocations, creating anti phase boundaries (APBs) in $\gamma^{\prime}$ precipitates is the active deformation mode and a source of superior creep strength [1-3]. Well above this temperature range, dislocation climb by-pass is usually found to be the primary deformation mode [4]. Multiple studies have found that in the intermediate temperature range (roughly between $650-750{ }^{\circ} \mathrm{C}$ ), following creep under a range of stresses, reordering processes such as microtwinning and isolated stacking faults become important factors on creep performance [5-8]. Koble [9] first hypothesized that the formation of microtwins involved the shear of Shockley partials pairs on adjacent $\{111\}$ planes in $\gamma^{\prime}$ precipitates, even though this would create a high energy two layer complex stacking fault (CSF). Kolbe proposed, without experimental evidence, that a reordering process must occur in the wake of the shearing partials, removing wrong nearest neighbor bonds. Kovarik et al. [4] using ab initio calculations found that not only was this reordering process energetically possible but multiple configurations could result in the subsequent low energy twin formation. Smith et al. [10] using the same reordering process described by Kovarik et al. [4] expanded it to include isolated superlattice extrinsic stacking fault (SESF) formation. In addition to reordering, Smith et al. [10] also reported that elemental segregation along faults and motion of a Cr- and Corich Cottrell atmosphere surrounding the shearing Shockley partials may be necessary for these shearing events to proceed through the $\gamma^{\prime}$ precipitates.

Another important deformation mechanism reported in this temperature range is the change from full dislocation motion in $\gamma$ channels to de-correlation, where full dislocations separate into their constituent Shockley Partials. Raujol et al. [11] found decorrelation to be active during creep at $700{ }^{\circ} \mathrm{C}$. In-situ analysis found that the de-correlated Shockley partials could proceed shearing both the $\gamma$ and $\gamma^{\prime}$ phases. Considering forces on both the shearing and trailing Shockley partials, Raujol et al. [11] found that channel width was an important factor in whether a dislocation would de-correlate or not. Later, Unocic et al. [12] hypothesized that de-correlation may be a precursor to microtwins as a source of the $\gamma^{\prime}$ shearing Shockley partials. They found decorrelated dislocations present in $\gamma$ channels in close proximity to microtwins after tensile tests conducted at $677{ }^{\circ} \mathrm{C}$ for ME3, a polycrystalline disk alloy currently used commercially.

\section{Orientation effects on Ni-based superalloys}

In polycrystalline alloys, grain-to-grain variations in the observed deformation modes not only depend on stress and temperature levels, but also how the individual grains are oriented relative to the loading axis. These differences between grains are not completely understood. It is possible that these differences reflect a significant dependence on Schmid factor; however, dissecting this dependence from those due to stress, temperature, and strain level is a formidable task. Furthermore, Phillips et al. [13] have shown that Schmid factors alone cannot accurately predict the active slip systems found in each grain. Low Schmid factor slip systems can become active during creep. It is not clear whether 
this is caused by local stresses found at interfaces like grain boundaries or if it is a grain-to-grain interaction effect.

Single crystal work by Kakehi et al. [14] determined that anisotropic creep properties are exhibited for single crystal alloys and an asymmetry is present depending on whether compressive or tensile load is applied. This variation in grain deformation mode makes creating accurate deformation models for predicting alloy creep performances a complex, yet scientifically rich, endeavor. In order to advance these efforts to understand and model the creep behavior of polycrystalline disk alloys, this study will employ single crystal surrogates to the disk alloys so as to create a more thorough understanding of the orientation, temperature, and stress dependence of deformation modes. The present paper reports on the behavior in two specific crystal orientations and focuses on two different alloy compositions. The deformation mechanism after modest creep strain is analyzed using advanced scanning transmission electron microscopy (STEM) based techniques. Of particular interest is how deformation varies and why between the different creep tests. In this paper, we focus on recent studies of specially-grown single crystals of two Ni-base superalloys, used in polycrystalline form for turbine engine disks. The single crystals enable exploration of the orientation and temperature effects on creep response, which is presently not known for this class of alloys. In addition, diffraction contrast STEM (DC-STEM) imaging is used to evaluate for the first time the operative deformation mechanisms in the intermediate temperature regime, spanning 700-800 ${ }^{\circ} \mathrm{C}$. Motivated by these experimental results, phase field modeling has been employed to study the interaction of matrix dislocations with realistic precipitate structures.

\section{Materials and Experimental Methods}

\section{$\underline{\text { Sample preparation }}$}

Single crystal analogs of two different disk alloys were obtained from GE Global Research Center; the commercial alloy ME3, and a modified alloy ME501 [15], with minor compositional changes. Table I below shows these differences in composition by weight percent.

Table I. Alloy compositions of ME3 and ME501

\begin{tabular}{|c|c|c|c|c|c|c|c|}
\hline Alloy & $\mathrm{Ni}$ & $\mathrm{Co}$ & $\mathrm{Cr}$ & $\mathrm{Mo}$ & $\mathrm{W}$ & $\mathrm{Nb}$ & $\mathrm{Ta}$ \\
\hline ME3 & Bal. & 20.6 & 13.0 & 3.8 & 2.1 & 0.9 & 2.4 \\
\hline ME501 & Bal. & 18.0 & 12.0 & 2.9 & 3.0 & 1.5 & 4.8 \\
\hline Alloy & $\mathrm{Al}$ & $\mathrm{Ti}$ & $\mathrm{Hf}$ & $\mathrm{C}$ & $\mathrm{B}$ & $\mathrm{Zr}$ \\
\hline ME3 & 3.5 & 3.4 & 0 & 0.05 & 0.03 & 0.03 \\
\hline ME501 & 3.0 & 3.0 & 0.4 & 0.05 & 0.03 & 0.05 \\
\hline
\end{tabular}

Both single crystal castings, which inherently have a level of chemical segregation greater than their powder metallurgy counterparts, underwent a heat treatment that formed a bi-modal $\gamma^{\prime}$ precipitate microstructure. To compare the resultant microstructures for both alloys, a sample from each alloy was polished down to a 1200 fine grit polish with a subsequent colloidal silica finish. After a $\gamma^{\prime}$ etchant consisting of $2 \mathrm{~mL}$ hydrofluoric acid, $30 \mathrm{~mL}$ nitric acid, and $50 \mathrm{~mL}$ lactic acid was applied the microstructure of both alloys were imaged using a FEI Sirion scanning electron microscope (SEM). A secondary detector was used over backscatter for improved surface resolution and to avoid subsurface precipitates from being included in the analysis. Figure 1 shows the differences between the two microstructures.

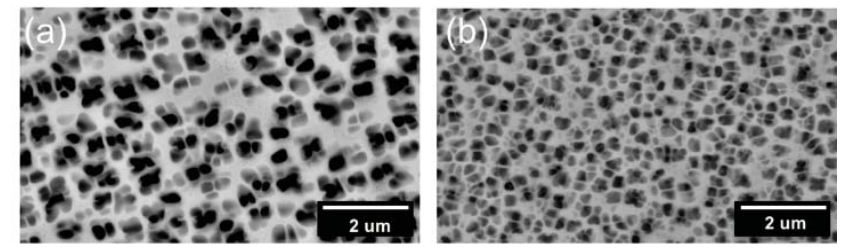

Figure 1. SEM backscatter image of (a) ME3 microstructure and (b) ME501 microstructure.

Some differences are observed in the microstructures of the two alloys, even though they experienced identical heat treatments and cooling rates. ME501 possesses a finer microstructure compared to ME3, while ME3 appears to have a coarser secondary $\gamma^{\prime}$ precipitates. To quantify these differences each microstructure, SEM images were analyzed using ImageJ [16]. Below Table II shows the differences in area fraction and precipitate size based on equivalent diameters between the two alloys.

Table II. Secondary and tertiary $\gamma^{\prime}$ precipitate area fraction and size in ME3 and ME501

\begin{tabular}{|c|c|c|c|c|}
\hline Alloy & $\begin{array}{c}\text { Secondary } \gamma^{\prime} \text { Area } \\
\text { Fraction (AF) }\end{array}$ & $\begin{array}{c}\text { Secondary } \\
\gamma^{\prime} \text { size }\end{array}$ & $\begin{array}{c}\text { Tertiary } \\
\gamma^{\prime} \text { AF }\end{array}$ & $\begin{array}{c}\text { Tertiary } \\
\gamma^{\prime} \text { size }\end{array}$ \\
\hline ME3 & $47 \%$ & $350 \mathrm{~nm}$ & $3-4 \%$ & $34 \mathrm{~nm}$ \\
\hline ME501 & $52 \%$ & $300 \mathrm{~nm}$ & $3-4 \%$ & $30 \mathrm{~nm}$ \\
\hline
\end{tabular}

\section{Creep Test Preparation}

Orientation Imaging Microscopy (OIM) was employed to determine the orientation for both alloy castings, as well as to detect and eliminate spurious grains from the regions used to prepare creep specimens. $7 \mathrm{~mm}$ long rectangular prism specimens were extracted for compression creep tests from each casting with a 1:1:2.5 dimensional ratio. Two orientations were extracted, [001] and [110], using electrical discharge machining (EDM). To remove the damage layer created by the EDM process, each side of the samples were polished down to a 1200 fine grit finish.

Three different temperature regimes were explored in this study: $700{ }^{\circ} \mathrm{C}, 760{ }^{\circ} \mathrm{C}$, and $815^{\circ} \mathrm{C}$. The stress varied from each test in an attempt to maintain a consistent strain rate at each temperature. For the ME501 samples, the compression samples were crept under $710 \mathrm{MPa}, 552 \mathrm{MPa}$, and $413 \mathrm{MPa}$, at each respective temperature. The ME3 samples were crept under these stresses as well; however, lower stresses were needed in order to keep strain rates comparable those displayed by the ME501 samples. Therefore, the stresses were reduced to $552 \mathrm{MPa}, 413 \mathrm{MPa}$ and $276 \mathrm{MPa}$, at each respective temperature. Each test was ended once about $0.5 \%$ plastic strain was reached. MTS 810 compression cages with attached linear variable displacement transducers (LVDTs) that recorded the displacement of the compression plattens were used to conduct each compression creep test. Thermocouples ( $\mathrm{K}$ type) were used to record the temperature for each test. Once the desired strain was reached, the 
test was immediately ended and the sample kept under load while being cooled rapidly under forced air. This procedure was an attempt to minimize changes in microstructure, chemistry and deformation as the sample reached room temperature.

\section{Deformation Characterization Methods}

After each compression test was completed a side of the compression cuboid was polished to remove any oxidation. Transmission electron microscope (TEM) foils were then extracted normal to the compression axis using an FEI Helios Nanolab Dualbeam 600 Focused ion beam microscope. By extracting the foils normal to the compression axis one last check could be made to ensure the sample was indeed cut to the right orientation. The TEM foils were analyzed using an FEI Tecnai F20 field emission $200 \mathrm{KV}$ STEM. STEM analysis was used in preference to conventional TEM (CTEM) for a multitude of reasons. An important one is the ability to image on low index zone axes which allows for multiple diffraction conditions to be activated at the same time. This allows for several slip systems to be imaged at the same time giving an improved overall image of the deformation modes active. Other advantages for using STEM include reduced bend contours and the ability to image thicker samples. A study of STEM diffraction contrast by Phillips et al. 17] found that the conventional $g \bullet b$ invisibility rules still apply for STEM and STEM images can be evaluated the same as CTEM images.

\section{Results}

\section{Compression Creep Response at $700^{\circ} \mathrm{C}$}

Figure 2(a) below shows the [001] and [110] oriented single crystal compression creep responses for ME3 and ME501 tested at $710 \mathrm{MPa}$. ME3 crept $1-2$ orders of magnitude faster than ME501 (with a strain rate of $5 \times 10^{-7} \mathrm{~s}^{-1}$ compared to ME501's $1 \times 10^{-9} \mathrm{~s}^{-1}$ strain rate). For better analysis on the deformation responses between the two alloys, two more ME3 tests were conducted using a lower stress of $552 \mathrm{MPa}$ to reach similar strain rates similar to those for ME501. At these slower strain rates, a prominent anisotropy in creep strength is observed, shown in Figure 2(b), with greater anisotropy between orientations experienced by the ME3 alloy.
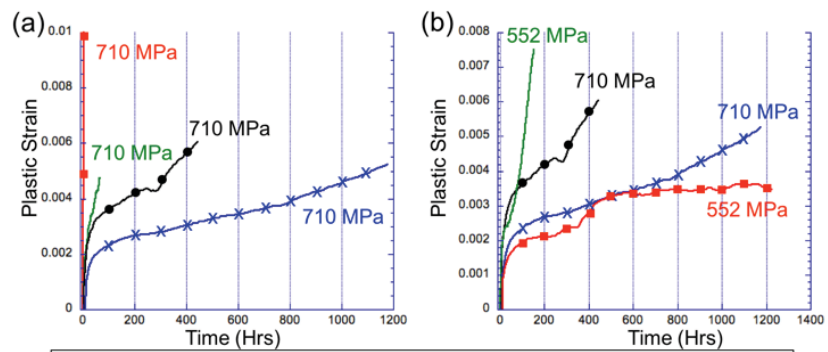

$$
\longrightarrow \text { ME3 [001] } \rightarrow \text { ME3 [110] } \rightarrow \text { ME501 [001] * ME501 [110] }
$$

Figure 2. $700{ }^{\circ} \mathrm{C}$ compression creep strain versus time curves from [001] and [110] orientated ME501 and ME3 samples with (a) same stress and (b) comparable strain rates.

For the comparable strain rate tests both alloys experienced the better creep strength in the [110] orientation. This coincides with reported compression creep strength values being considerably lower for [001]-oriented crystals than for other orientations $[14,18]$. The poor compression creep performance in this orientation has been attributed to events that cause local stress relaxation, such as $\gamma^{\prime}$ shearing by microtwins or SESFs [19-21]. Additionally, the [001] orientation has the largest number of active slip systems of any orientation, allowing for the activation of more glide systems initially, and resulting in the observed high primary strain rates [22]. Fluctuations in the creep curves are most likely a result of the very low strain rates that are approaching the resolution limit of the LVDT's. These small instabilities are believed to be the result of small temperature fluctuations in the furnace and/or other external variables that become apparent in these very low strain rate creep tests.

\section{Compression Creep response at $760{ }^{\circ} \mathrm{C}$}

At increased temperature of $760{ }^{\circ} \mathrm{C}$, the stress levels were lowered to $552 \mathrm{MPa}$ in order to maintain strain rates near $1 \times 10^{-9} \mathrm{~s}^{-1}$. As shown in Figures 3(a) and 3(b), similarities in the creep responses were seen in this temperature regime when compared with 700 ${ }^{\circ} \mathrm{C}$. Once again, ME3 crept at rates significantly faster than ME501 at the same applied stress. Once again additional tests were performed to achieve comparable strain rates, as shown in Figure $3 b$.
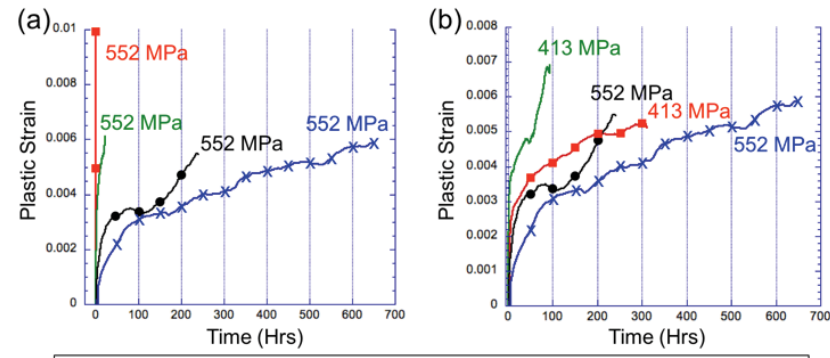

$\longrightarrow \operatorname{ME3}[001] \longrightarrow \operatorname{ME3}[110] \longrightarrow \operatorname{ME501}$ [001] $\rightarrow$ ME501 [110]

Figure 3. $760{ }^{\circ} \mathrm{C}$ compression creep strain versus time curves from [001] and [110] orientated ME501 and ME3 samples with (a) same stress and (b) comparable strain rates.

Though the anisotropy appears to be reduced compared to the 700 ${ }^{\circ} \mathrm{C}$ tests, the creep curves again have different shapes depending on the orientation. For the [001] orientations, the curve revealed a small amount of strain hardening followed by accelerating creep strain rates. In contrast, the [110] oriented crystals revealed continual hardening as the tests progressed. In this case, ME501 performed better, even when ME3 was crept under the reduced stress.

\section{Compression Creep response at $815^{\circ} \mathrm{C}$}

The next set of compression creep tests were performed at $815^{\circ} \mathrm{C}$ and $413 \mathrm{MPa}$. The ME3 alloy predictably exhibited reduced strength, relative to ME501, and new tests were carried out with a stress of $276 \mathrm{MPa}$ to obtain comparable strain rates. Figure 4 below reveals the creep curves for all the tests at $815^{\circ} \mathrm{C}$. At 815 ${ }^{\circ} \mathrm{C}$, the anisotropy between [001] and $\{110\}$ orientations that was observed at $700{ }^{\circ} \mathrm{C}$ and $760{ }^{\circ} \mathrm{C}$ has become much smaller, no orientation seems to show superior creep strength over the other. However, ME501 still possesses clearly superior creep strength compared to ME3 when both were crept at $413 \mathrm{MPa}$. 

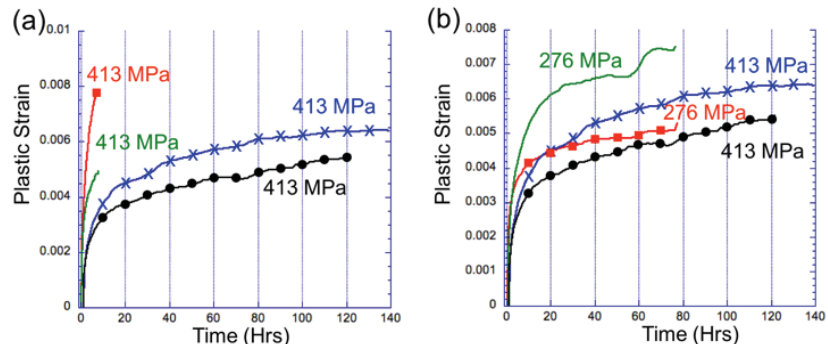

$\longrightarrow$ ME3 [001] $\longrightarrow$ ME3 [110] $\longrightarrow$ ME501 [001] $\rightarrow$ ME501 [110]

Figure 4. $815^{\circ} \mathrm{C}$ compression creep curves from [001] and [110] orientated ME501 and ME3 samples with (a) same stress and (b) comparable strain rates.

\section{Compression Creep Response: Polycrystalline vs. Single Crystal}

In an effort to improve understanding of grain boundary effects on creep strength, new compression tests were conducted on polycrystalline ME501 specimens of similar size. Below in Figure 5 is the comparison between the polycrystalline creep response to that of the ME501 single crystals at (a) $700{ }^{\circ} \mathrm{C}$ and (b) $760{ }^{\circ} \mathrm{C}$.
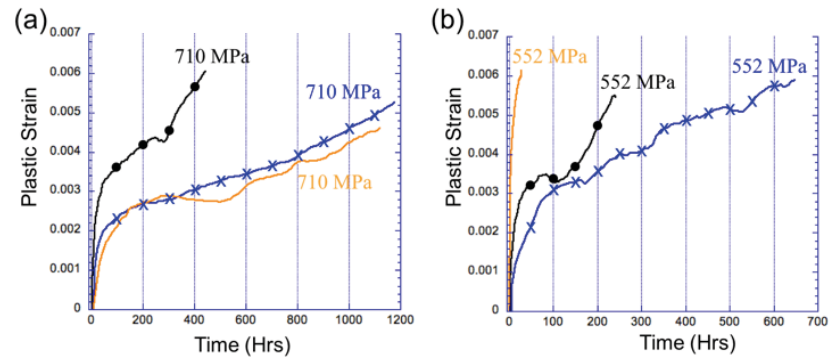

$\longrightarrow$ ME501 Polycrystal—- ME501 [001] — ME501 [110]

Figure 5: Compression creep strain versus time curves from [001] [110] orientated and polycrystalline ME501 (a) at $700{ }^{\circ} \mathrm{C}$ and (b) at $760{ }^{\circ} \mathrm{C}$.

\section{Deformation Analysis Using DC-STEM for $700{ }^{\circ} \mathrm{C}$ tests}

Due to space only the samples crept at the target strain rate of $10^{-9}$ $\mathrm{s}^{-1}$ will be discussed presently for the STEM deformation analysis. Representative, zone axis bright field STEM (BF-STEM) deformation analysis images are shown in Figures $6 \mathrm{a}$ and $6 \mathrm{~b}$ for ME3 and ME501, respectively, and both orientations after $700{ }^{\circ} \mathrm{C}$ compression creep tests. Despite the alloying and microstructure differences observed in the two alloys, both ME3 and ME501 exhibited similar deformation modes. For the [110] orientation, which possessed superior creep strength, STEM diffraction contrast reveals full dislocation activity in the $\gamma$ channels and stacking fault ribbons active for both alloys. Stacking fault ribbons are usually observed in [001] single crystals pulled in tension and require the interaction of unlike $1 / 2<110$ 's $>[23,24]$. Another important observation is the intrinsic stacking faults (ISFs) in the $\gamma$ channels found in both the ME3 and ME501 in the [001] orientation. The presence of these stacking faults implies that de-correlation of the $1 / 2<110>$ full dislocations into their constituent $1 / 6<112>$ Shockley partials is active. As well as decorrelation, shearing events such as isolated SESFs and microtwins are present in the [001] orientation for both alloys. The difference in deformation modes between the two alloys correlates with the anisotropy observed in the creep curves between the [001] and [110] orientations.

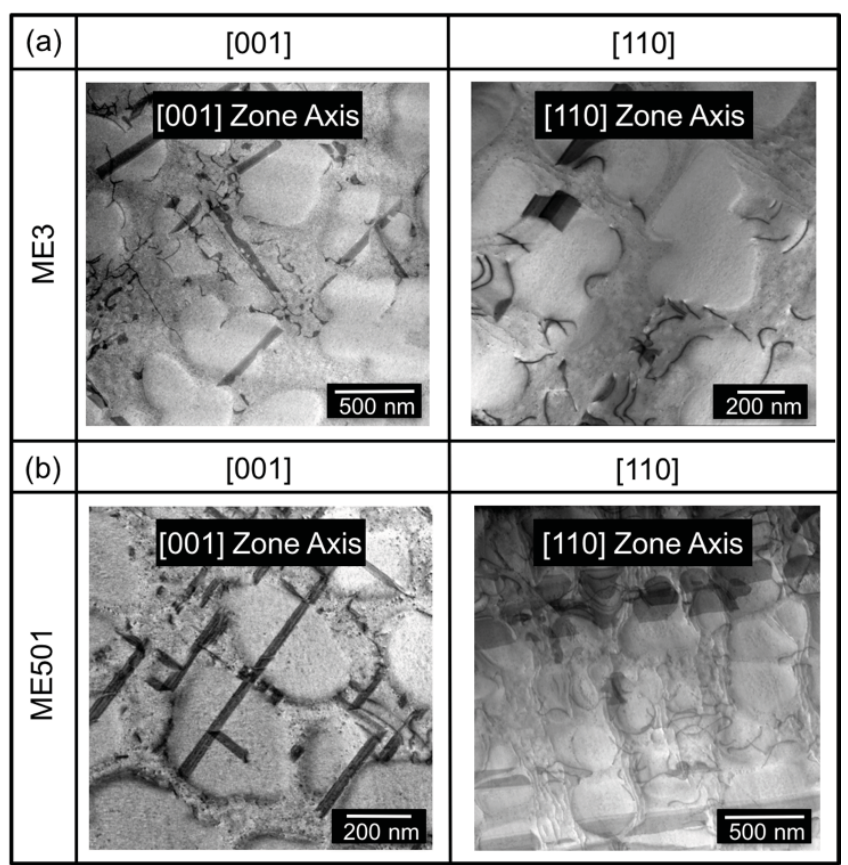

Figure 6. BF-STEM Zone Axis images for both [001] and [110] orientations after $700{ }^{\circ} \mathrm{C}$ compression creep tests for (a) ME3 and (b) ME501.

\section{Deformation Analysis Using DC-STEM for $760{ }^{\circ} \mathrm{C}$ tests}

Figure 7 shows examples of the primary deformation modes observed from ME501 and ME3 specimens after compression creep tests at $760{ }^{\circ} \mathrm{C}$.

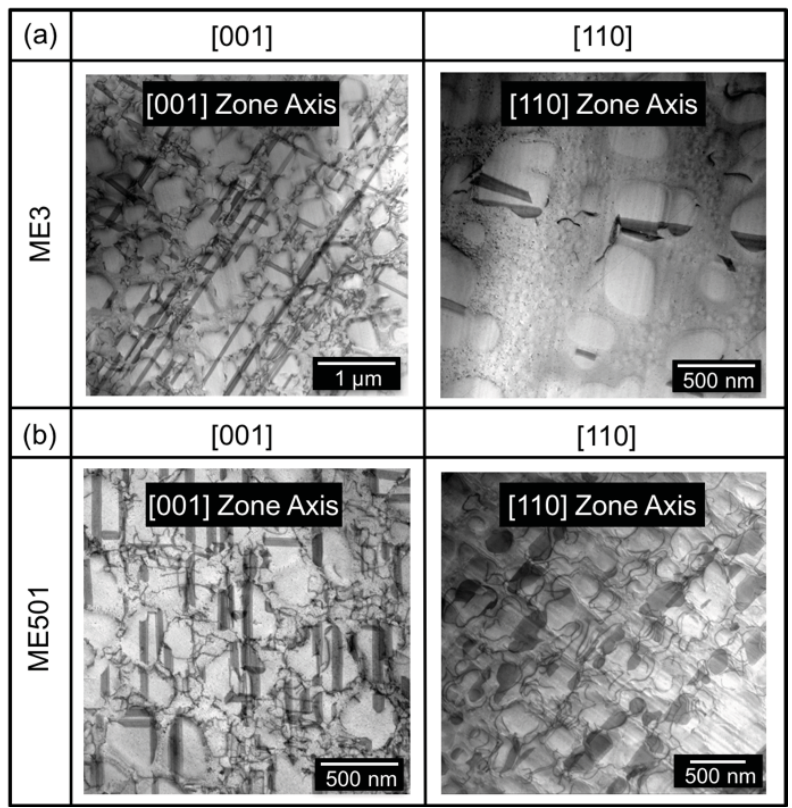

Figure 7. BF-STEM Zone Axis images for both [001] and [110] orientations after $760{ }^{\circ} \mathrm{C}$ compression creep tests for (a) ME3 and (b) ME501. 
There is no evidence of ISFs in the $\gamma$ channels, indicating that decorrelation is no longer observed at $760{ }^{\circ} \mathrm{C}$ in [001] samples. Instead of dislocation de-correlation, numerous $\gamma^{\prime}$ shearing events are active; however, an apparent difference in the type of shearing events exists between ME3 and ME501. For ME3, the long faults that extend through both the $\gamma$ and $\gamma^{\prime}$ phases are microtwins, confirmed using high angle annular dark field (HAADF) imaging [25]. The presence of numerous microtwins in ME3, reveals that the hypothesis by Unocic et al. [12] that dislocation de-correlation is necessary for microtwin formation may not be exclusively correct. Indeed, other unknown dislocation interactions and pathways must exist that can result in microtwin formation. In the ME501 specimen, only isolated SESFs are present. The formation of these SESF's was discussed in full by Smith et al. Again for the [110] orientations in both alloys full dislocation activity in the $\gamma$ channels and stacking fault ribbons were active.

\section{Deformation Analysis Using DC-STEM for $815^{\circ} \mathrm{C}$ tests}

Figure 8 shows the active deformation modes observed in ME501 and ME3 specimens after compression creep tests at $815{ }^{\circ} \mathrm{C}$. Interestingly, as the anisotropy between the two different orientations disappeared in the creep curves at $815{ }^{\circ} \mathrm{C}$ so did the difference in deformation mechanisms. For each alloy at both orientation, climb by-pass becomes the dominant mechanism for creep tests at $815^{\circ} \mathrm{C}$ while $\gamma^{\prime}$ shearing events were significantly reduced in frequency.

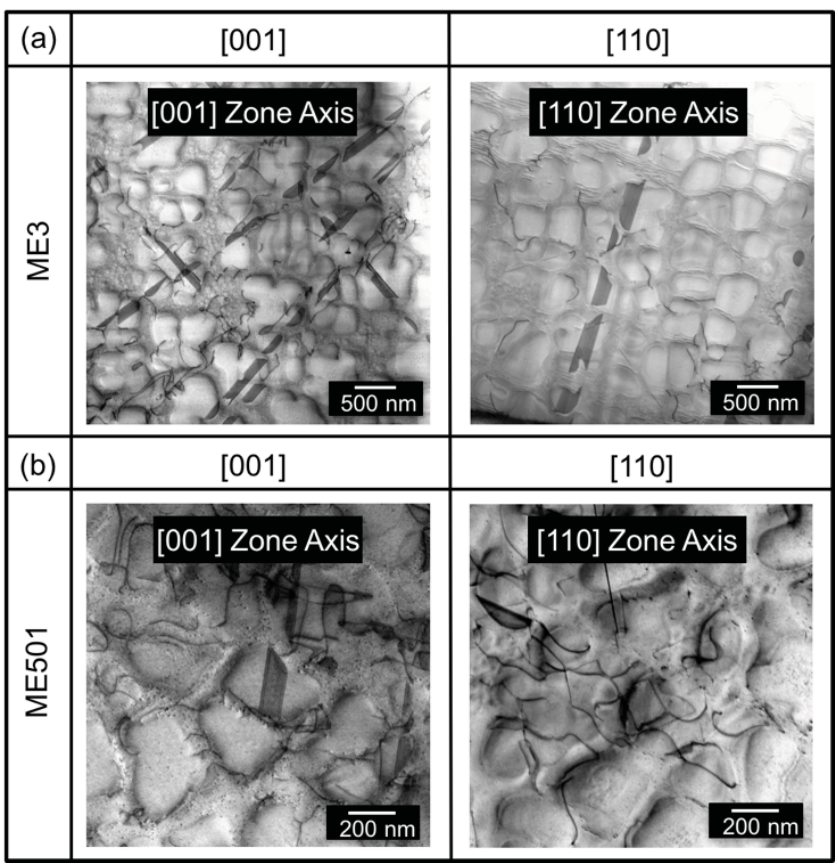

Figure 8. BF-STEM Zone Axis images for both [001] and [110] orientations after $815{ }^{\circ} \mathrm{C}$ compression creep tests for (a) ME3 and (b) ME501.

\section{Discussion}

\section{Orientation Effects on Deformation}

A relationship between the macro-scale creep behavior and nanoscale deformation is clearly present. The creep anisotropy between the [110] and [001] orientations at $700{ }^{\circ} \mathrm{C}$ and $760{ }^{\circ} \mathrm{C}$ correlates very well with differences in active shearing mechanisms.

For the [110] orientations in both alloys, stacking fault ribbons were prominent, while full dislocations were found in the $\gamma$ matrix. A stacking fault ribbon is usually observed amid multiple adjacent $\gamma^{\prime}$ precipitates to avoid the high energy APBs. This leaves the constituent $1 / 2<112>$ dislocations separated by perfect crystal, where the latter forms a SESF. Re-ordering must take place for the formation of the SESF and while reordering is not necessary for the SISF, it may need to occur if the $1 / 3<112>$ super-partials have dissociated [26]. These reordering mechanisms are likely rate-controlling and may slow the ability for the stacking fault ribbon to shear. Multiple studies have reported stacking fault ribbons to be the source of superior creep strength in [001] single crystals crept in tension [23,26-28]. For the [001] samples, both ME3 and ME501 experienced similar active deformation modes. At $700{ }^{\circ} \mathrm{C}$, the presence of microtwins in ME3 and isolated stacking faults in ME501 were observed as well as de-correlated dislocations in the $\gamma$ matrix. This is the only time dislocation de-correlation is observed in both ME3 and ME501. All other cases only revealed the presence of full dislocation activity in the channels. For both ME3 and ME501, the frequency of $\gamma^{\prime}$ shearing events is significantly increased at $760{ }^{\circ} \mathrm{C}$ compared to $700{ }^{\circ} \mathrm{C}$. Again, microtwins were found in ME3 while isolated stacking faults were observed in the ME501 sample.

For the $815{ }^{\circ} \mathrm{C}$ compression creep experiments, the different $\gamma^{\prime}$ shearing events and exclusively channel-bound, full $1 / 2[110]$ dislocations are replaced with climb by-pass of the full dislocations for all orientations and alloys. Two reasons for this change in deformation mode could be the increased temperature during the creep tests providing more energy for dislocation climb, and the dissolution of the tertiary $\gamma^{\prime}$ precipitates in the channels. As expected, the change in active deformation modes to climb by-pass for all specimens correlates with the disappearance of anisotropy in the creep curves at $815{ }^{\circ} \mathrm{C}$, supporting the hypothesis that creep anisotropy is directly related to the type of deformation mode active between orientations. From these results, a qualitative ranking of $\gamma^{\prime}$ shearing modes, from best to worst, can be assembled and is shown below with stacking fault ribbons promoting superior creep properties:

$$
\text { Stacking Fault Ribbons }>\text { Isolated SESFs }>\text { Microtwinning }
$$

This ranking is further supported by the work of Rae et al .[27] who found stacking fault ribbons to be the cause of superior creep strength in [001] single crystals crept in tension, in addition to work by Yamashita et al. [21] who found microtwin formation correlates with poor creep strength. . On the other hand, Unocic, et al. [29] have compared the creep response in tension for polycrystals of fine (rim) versus course (bore) microstructures in $\mathrm{ME} 3$ at $677{ }^{\circ} \mathrm{C}$, that the former exhibited microtwinning and better creep strength than the latter at the same stress level. However, trying to correlate deformation to creep response in polycrystals is difficult due to local and grain to grain variations in observed deformation. Without a rigorous statistical study, any conclusion will be confounded by local grain boundary effects and other unaccounted variables. 


\section{Dislocation Activity Diagram (DAD) - Predicting De-Correlation}

The dislocation movement through the $\gamma$ matrix channel is one of the major factors determining the creep mechanisms in Ni-based superalloys. When dislocation sources are distributed throughout the microstructure, the $\gamma$-channel width controls the characteristics of the looping mechanisms (full looping, partial looping, or unable to loop). This has been investigated in previous works [12], where an analytical DAD model has been developed to investigate the critical shear stresses of full and partial (decorrelated) dislocation looping. The activation conditions for decorrelation and full dislocation looping have been found to be sensitive to both precipitate microstructure (especially $\gamma$-channel width) and other physical parameters such as shear modulus, lattice friction and work hardening in the channel. At the same time, it has been observed that de-correlation occurs at lower stress levels relative to other possible processes (for example, full dislocation Orowan looping and APB shearing). For decorrelation to occur, the direction of applied shear stress must be such that sufficient stress magnitude is applied on the leading Shockley partial [11].

The dislocation sources in these supersolvus heat treated alloys are sparse, usually associated with intragranular carbides or grain boundary sources in polycrystals, as the initial dislocation density is very small. In this case, the operating deformation mechanism can only be revealed as a result of dislocation percolation, when the dislocations are initiated from the rare sources. In this work, a percolation simulation approach is designed based on the analytic DAD diagram and direct SEM observation of the precipitate microstructure. In this approach, the critical channel widths of dislocation activation must first be calculated by the DAD for a given combination of physical parameters such as shear modulus, lattice friction and work hardening. In the DAD, critical loading conditions for dislocation activation are represented by boundaries of stress magnitude and direction. The critical channel width is obtained when the external loading condition falls on a boundary shown in the DAD, where fulfillment of the following equations occurs:

$$
\begin{aligned}
& \tau_{\mathrm{crt}}(\mathrm{ChW})=\sigma_{\mathrm{app}} \\
& \phi_{\mathrm{crt}}(\mathrm{ChW})=\theta_{\mathrm{app}}
\end{aligned}
$$

Where $\mathrm{ChW}$ is channel width, $\tau_{\text {crt }}$ is magnitude of critical stress and $\phi_{\text {crt }}$ is the direction, $\sigma_{\text {app }}$ is the magnitude of applied stress and $\theta_{\text {app }}$ is the direction. When both the equations are satisfied, they provide the solution of $\mathrm{ChW}$ which gives the critical channel width where de-correlation is promoted. In order to apply the concept of the DAD on an actual microstructure, it is insufficient to consider only average microstructure parameters. For instance, a dislocation segment will pass through the matrix continuously until it meets a channel narrower than the critical value. Therefore, a percolation study is needed to determine the critical channel width. In the percolation simulation, a dislocation line is set to proceed from some unknown source and into a precipitate microstructure obtained directly from segmented SEM images. The SEM images are obtained in $\langle 111\rangle$ oriented grains such that the precipitate cross-sections are most accurate to that sampled by dislocations on a $\{111\}$ shear plane. Finally, the sheared area-fraction and percolation distance are obtained by the simulation, and the activation probability of each deformation mode is estimated.

\subsubsection{Determining deformation mechanism populations}

Using the input parameters in Table III, the critical channel widths for full dislocation activity and for de-correlation are calculated to be $156 \mathrm{~nm}$ and $117 \mathrm{~nm}$, respectively.

Table III. The input parameters from the DAD calculation

\begin{tabular}{|l|l|}
\hline Intrinsic stacking fault energy & $10 \mathrm{~mJ} / \mathrm{m}^{2}[11]$ \\
\hline Shockley partial Burgers vector & $\mathrm{b}=0.146 \mathrm{~nm}[1]$ \\
\hline Shear modulus & $\mu=67.2 \mathrm{E} 3 \mathrm{MPa}[1]$ \\
\hline Lattice friction & $f=15 \mathrm{MPa}\left(700{ }^{\circ} \mathrm{C}\right)[30]$ \\
\hline Line tension & $\alpha=1.5[1]$ \\
\hline Secondary $\gamma^{\prime}$ area fraction & $47.2 \%$ \\
\hline Tertiary $\gamma^{\prime}$ area fraction & $3.5 \%$ \\
\hline Applied stress & $552 \mathrm{MPa}$ along [001] direction \\
\hline
\end{tabular}

Due to the distribution in channel widths in the microstructure, there are certain channels (white area in Figure 9) that allow activation of only the de-correlation mode. Therefore, locally the deformation microstructure will be dominated by de-correlation that will spread throughout until encountered by obstacles. An example of the percolation simulations, performed for a given microstructure with dislocations entering from the 4 edges is shown in Figure 10. In each case, a full dislocation is placed initially on the edge and an applied load drives the dislocation into the microstructure. The white colored region represents the full dislocation sheared region, which is bounded by narrower channels. These narrower channels allow only the leading partial dislocation to pass through. As a result, de-correlation is activated in the pink colored area. Finally, much narrower channels prohibit movement by any kind of dislocation, which results in unsheared regions (red colored areas).

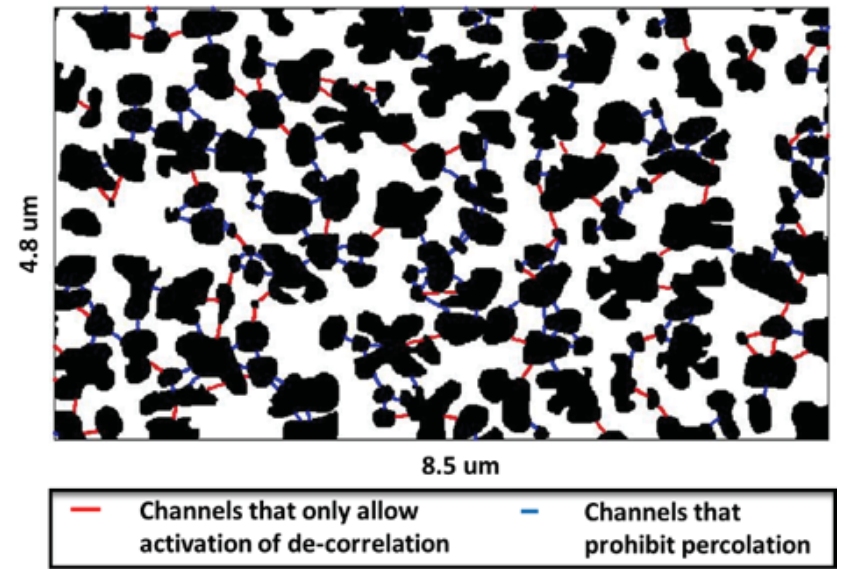

Figure 9. Channels in a ME3 precipitate microstructure. The secondary precipitate cross-sections are shown in black. The average channel width is $210 \mathrm{~nm}$. The critical channel width for the activation of de-correlation (red) is $156 \mathrm{~nm}$, while that for stopping even partial looping (blue) is $65 \mathrm{~nm}$. Please refer to pdf. file in the conference $\mathrm{CD}$ for the colored version of this figure.

The average area fraction of full dislocation shearing is calculated to be $27.4 \%$, while the average area fraction of partial dislocation shearing (de-correlation activation) is calculated to be $64.3 \%$. In addition, the average percolation distance (measured from each edge) of full dislocations is only $\sim 3.0 \mu \mathrm{m}$. Thus the fraction of full 
dislocation activation is expected to be only concentrated in the vicinity of their sources. According to these percolation simulations, the de-correlation area dominates for the given microstructure and loading condition, in spite of a rather broad channel width distribution in the microstructure.

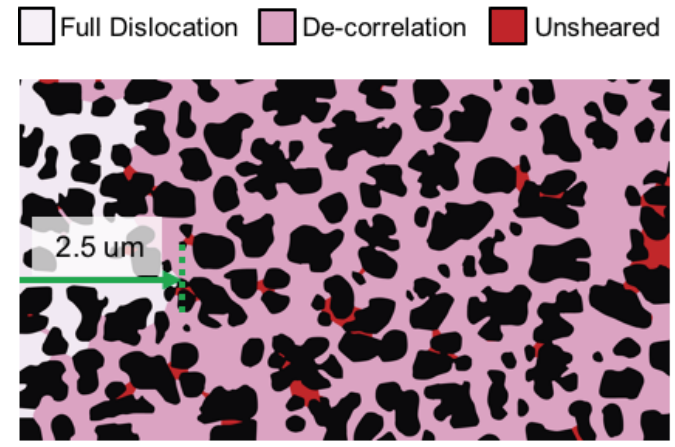

Full dislocation: $14.5 \%$; De-correlation: $80.4 \%$

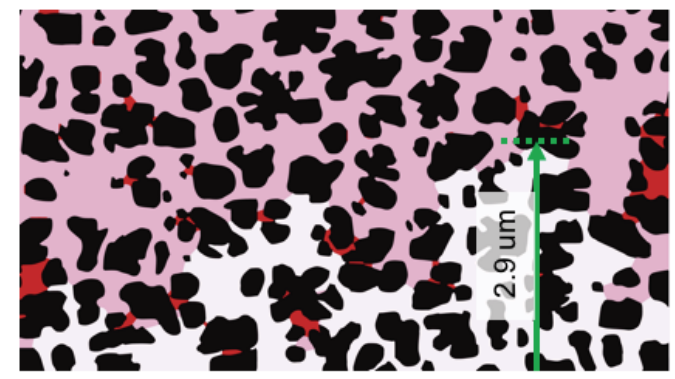

Full dislocation: $33.8 \%$; De-correlation: $60.5 \%$

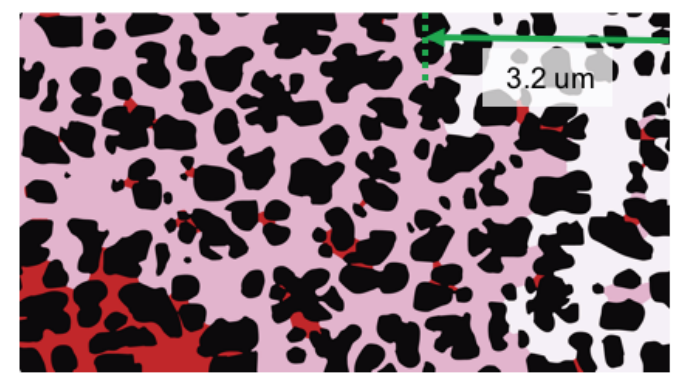

Full dislocation: $22.5 \%$; De-correlation: $63.7 \%$

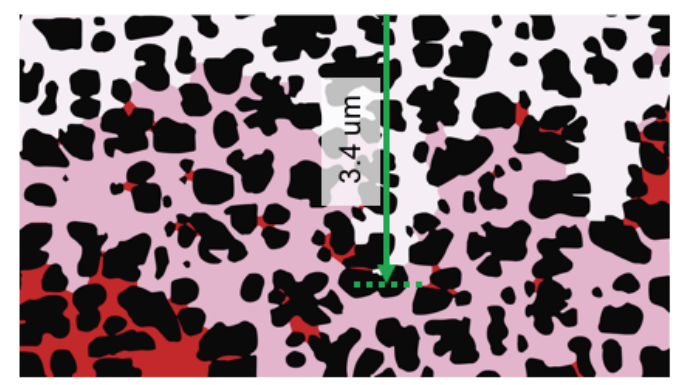

Full dislocation: 36.0\%; De-correlation: $52.4 \%$

Figure 10. Percolation simulations preformed when dislocations are initiated at grain boundaries. white color indicates a closed zone of full dislocation activation. pink color shows the activation area of de-correlation. The red color shows unsheared area. Percolation distances are indicated by the green arrows.
It should be emphasized that it is the critical channel width rather than the average channel width that determines the active deformation modes, as demonstrated clearly by the above percolation simulations. Thus, critical channel width rather than average channel width should be used in DAD analysis. For the microstructure shown in Figure 9, for example, if the average channel width of $210 \mathrm{~nm}$ is used, then the DAD calculation incorrectly predicts full dislocation activity at $700{ }^{\circ} \mathrm{C}$ in both the [110] and [001] orientations at $552 \mathrm{MPa}$ as shown in Figure 11(a).

This does not match the experimental findings of de-correlation in the [001] orientation at $700{ }^{\circ} \mathrm{C}$. Additionally, the simulations in Figure 10 found that for the [001] orientation the smaller channel widths controlled the majority of the deformation by forcing full dislocations to de-correlate in order to propagate further. As expected, when the critical channel width for the activation of decorrelation $(156 \mathrm{~nm})$ is used, as shown in Figure 11(b), then the DAD predicts de-correlation as the active deformation mode in the [001] oriented sample, whereas full dislocation as the active deformation mode for the [110] oriented sample. This matches the deformation microstructures observed experimentally for both ME3 and ME501 at this temperature. Therefore, when a wide range of channel width values are present in a given microstructure, the DAD analysis should be used in combination with the percolation simulation in order to make an accurate prediction of dislocation activity.

(a)

\section{Channel Width $=210 \mathrm{~nm}$}

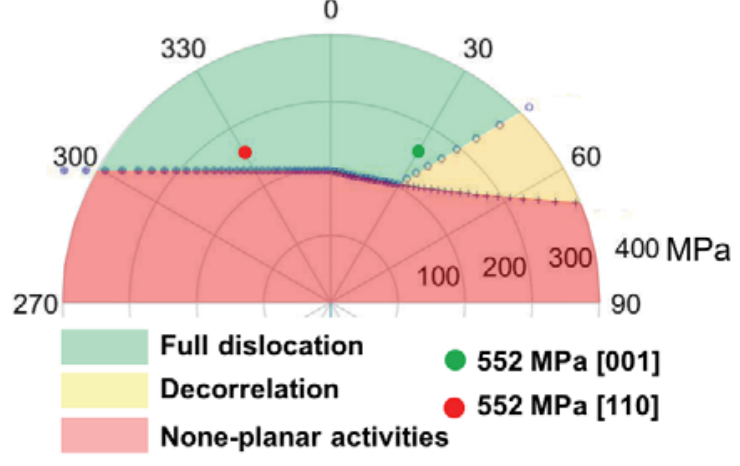

(b)

$$
\text { Channel Width }=156 \mathrm{~nm}
$$

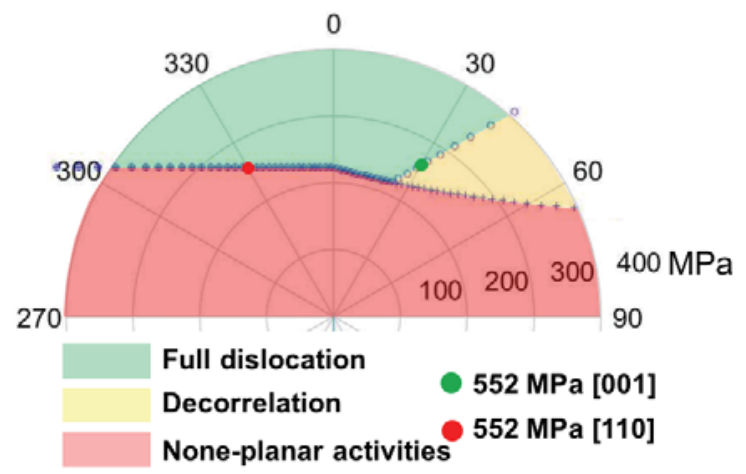

Figure 11. DAD is calculated by using (a) average channel width $(210 \mathrm{~nm})$ and (b) the critical channel width for de-correlation (156 $\mathrm{nm})$. 


\section{Ni-base Disk Alloy Deformation Mechanism Map}

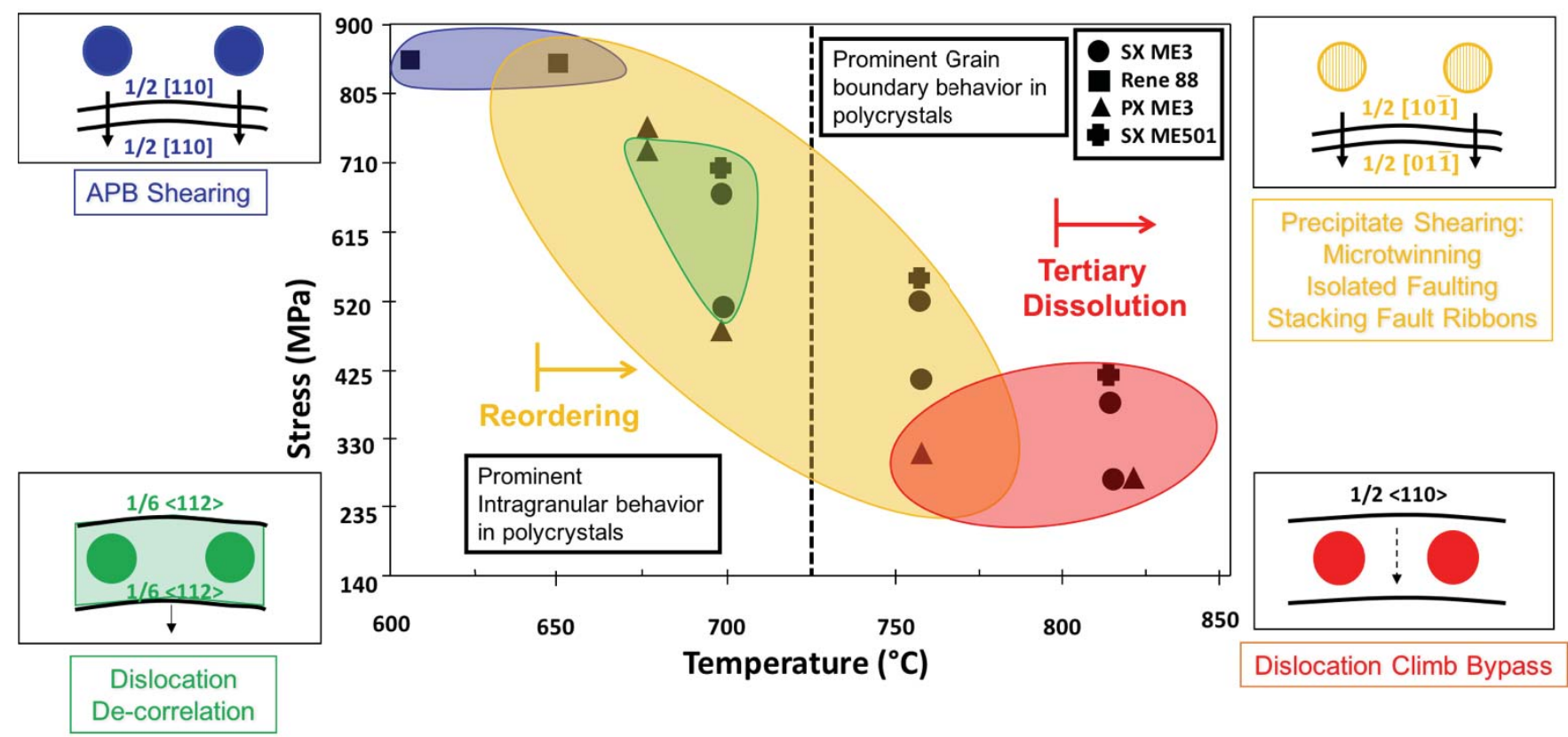

Figure 12. Deformation mechanism map for Ni-base disk alloys at varying temperature and stresses $[1,5,6]$.

\section{$\underline{\text { Mechanism Map }}$}

Combining the findings in this study with those mentioned in the introduction, a mechanism map (Figure 12) inspired by Unocic [31], can be created for polycrystalline disk alloys to help predict the type of deformation modes in different stress and temperature regimes. The mechanism map above reveals four main deformation zones; athermal shearing (APB shearing), thermally activated shearing (microtwinning, isolated stacking faults, and stacking fault ribbons), dislocation de-correlation, and dislocation climb bypass. Although precipitate shearing is considered one zone, it comprises related, but distinct, mechanisms such as microtwinning, isolated faulting, and stacking fault ribbons. These shearing modes all share similar characteristics such as the shearing of secondary $\gamma^{\prime}$ precipitates by Shockley partials and the requirement for reordering, yet the formation of these mechanisms involves different dislocation interactions and processes. Consequently, they are very sensitive to grain orientation and $\gamma^{\prime}$ microstructure and volume fraction. For example, at $760{ }^{\circ} \mathrm{C}$ all three precipitation shearing modes were active depending on the stress, orientation, and composition of the alloy. The effect of composition at this temperature range has been further explored by Smith et al. [25] regarding the differences observed between ME3 and ME501. They found that an increase in $\eta$ phase formers $(\mathrm{Nb}, \mathrm{Ta}$, and $\mathrm{Ti})$ inhibited the formation of twins and instead promoted isolated SESFs formation.

Dislocation de-correlation appears to be very temperature and stress dependent. Indeed, de-correlation appears only in a small window of stress and temperature around $700{ }^{\circ} \mathrm{C}$. Moreover, decorrelation appears to favor orientations at or near $<001>$ as was observed in this study. As was discovered in the ME501 polycrystalline creep tests at around $725{ }^{\circ} \mathrm{C}$ grain boundary behavior, such as grain boundary sliding, becomes the dominant creep mechanism, significantly reducing creep strength for polycrystals above this temperature. Another observation from the creep tests was the dissolution of tertiary $\gamma^{\prime}$ precipitates after the $815{ }^{\circ} \mathrm{C}$ tests. For example, tertiary $\gamma^{\prime}$ precipitates in ME501 were found to dissipate from $3.3 \%$ to $.4 \%$ area fraction after the [001] $815^{\circ} \mathrm{C}$ creep test. In addition to the increase in temperature, this tertiary $\gamma^{\prime}$ dissolution may help explain the change in deformation mechanism from precipitate shearing to climb bypass. Lastly, at high stress low temperature tests APB shearing is clearly dominate.

\section{Conclusions}

This study explored the effects orientation and alloying have on deformation activity and the subsequent macro-scale creep behavior in Ni-base disk alloys. Multiple single crystal compression creep tests were conducted in different stress and temperature regimes for two different orientations; [001] and [110] for ME3 and ME501. From these tests and the following STEM deformation analysis the following conclusions can be made.

(i) In compression creep the [110] orientation is superior to the [001] orientation due to the formation of stacking fault ribbons in [110] and microtwinning/isolated faulting in [001] orientations.

Above $815^{\circ} \mathrm{C}$ the creep anisotropy between the two orientations disappears along with differences in active deformation. All deformation analysis found climb bypass active at this temperature.

(iii) Dislocation de-correlation is a unique deformation mode found in a small temperature and stress regime and only 
in favorable orientations. Dislocation activity diagram calculations found channel widths to be a significant factor in whether dislocations will de-correlate in the $\gamma$ channels.

Polycrystals creep at comparable rates to single crystals of ME3 at $700{ }^{\circ} \mathrm{C}$, but much faster than single crystals at $760{ }^{\circ} \mathrm{C}$, indicating the onset of grain boundary dominated creep behavior in this temperature range.

(v) A mechanism map, using data from this study and past work, has been created to help predict the type of deformation that will be active in different stress and temperature regimes.

In summary, these results prove grain orientation and alloy composition have a profound effect on deformation modes and subsequent creep behavior and these effects need to be incorporated into future modelling efforts.

\section{Acknowledgements}

MJM and YW acknowledge the support of NSF and the DMREF program under grant \# 1534826. TMS and DL acknowledge the support of GE Aviation for their support of this work through the GE University Strategic Alliance (USA) program. TMS acknowledges Lee Casalena for helping create and format figures.

\section{References}

1. R. R. Unocic et al., "Mechanisms of creep deformation in polycrystalline Ni-base disk superalloys," Material Science and Engineering A, 483-484 (2008), 25-32.

2. Y. Yuan et al., "Influence of Co content on stacking fault energy in Ni-Co base disk superalloys," Journal of Mateials. Reearch, 26 (2011), 2833-2837.

3. E. S. Huron et al., "Controlling the deformation mechanism in disk superalloys at low and intermediate temperatures," Superalloys 2012, (2102), 35-42.

4. L. Kovarik et al., "Microtwinning and other shearing mechanisms at intermediate temperatures in Ni-based superalloys," Progress in Material Science, 54 (2009), 839-873.

5. G. B. Viswanathan et al., "Microtwinning during intermediate temperature creep of polycrystalline Ni-based superalloys: mechanisms and modelling," Philosophical Magazine, 86 (2006), 4823-4840.

6. H. Z. Deutchman et al., "Deformation mechanisms coupled with phase field and crystal plasticity modeling in a high temperature polycrystalline ni-based superalloy," Superalloys 2012 (12th Int. Symp. Superalloys), (2012) 25-33.

7. L. Kovarik, et al., "The intermediate temperature deformation of ni-based superalloys : importance of reordering," JOM, 61 (2009), 42-48.

8. S. Karthikeyan et al., "Modeling microtwinning during creep in Ni-based superalloys," Scripta Materialia, 54 (2006) 1157-1162.
9. M. Kolbe, "The High Temperature Decrease of the Critical Resolved Shear Stress in Nickel-Base Superalloys," Material Science and Engineering, (2001), 383.

10. T. M. Smith et al., "Segregation and $\eta$ phase formation along stacking faults during creep at intermediate temperatures in a Nibased superalloy," Acta Materialia, 100 (2015), 19-31.

11. S. Raujol et al., "Decorrelated movements of Shockley partial dislocations in the $\gamma$-phase channels of nickel-based superalloys at intermediate temperature," Philosophical Magazine, 86 (2006), $1189-1200$.

12. R. R. Unocic et al., "Dislocation Decorrelation and Relationship to Deformation Microtwins During Creep of a $\gamma$ ' Precipitate Strengthened Ni-Based Superalloy," Acta Mater., 59 (2011), 7325-7339.

13. P. J. Phillips et al., "Low cycle fatigue of a Ni-based superalloy: Non-planar deformation," Scripta Materialia, 62 (2010), 790-793.

14. K. Kakehi, "Tension/compression asymmetry in creep behavior of a Ni-based superalloy," Scripta Materialia, 41 (1999), $461-465$.

15. K. R. Bain et al., "United States Patent,” US 8.992,699 B2, (2015).

16. M. D. Abramoff, P. J. Magalhaes, and S. . Ram, "Image Processing with ImageJ," Biophotonics Int., 11 (2004) no. 7, 3644.

17. P. J. Phillips et al., "Diffraction contrast STEM of dislocations: Imaging and simulations," Ultramicroscopy, 111 (2011), 1483-1487.

18. K. Kakehi, "Effect of Plastic Anisotropy on the Creep Strength of Single Crystals of a Nickel-Based Superalloy," Metallurgcal Transactions A, 31 (2000), 421-430.

19. C. M. F. Rae and R. C. Reed, "Primary creep in single crystal superalloys: Origins, mechanisms and effects," Acta Materialia, 55 (2007), 1067-1081.

20. R. Srinivasan, G. F. Eggeler, and M. J. Mills, "Gamma Prime cutting as rate-conrolling recovery process during hightemperature and low-stress creep of superalloy single crystals," Acta Materialia, 48 (2000), 4867-4878.

21. M. Yamashita and K. Kakehi, "Tension/compression asymmetry in yield and creep strengths of Ni-based superalloy with a high amount of tantalum," Scripta Materialia, 55 (2006), 139-142.

22. D. M. Knowles and Q. Z. Chen, "Superlattice stacking fault formation and twinning during creep in gamma/gamma prime single crystal superalloy CMSX-4," Material Science and Engineering A, 340 (2003), 88-102.

23. V. A. Vorontsov et al., "Shearing of $\gamma$ ' Precipitates by a $<112>$ Dislocation Ribbons in Ni-Base Superalloys: A Phase Field 
Approach,” Acta Materialia, 58 (2009), 4110-4119.

24. C. M. F. Rae, N. Matan, and R. C. Reed, "The role of stacking fault shear in the primary creep of [001]-oriented single crystal superalloys at $750^{\circ} \mathrm{C}$ and $750 \mathrm{MPa}$," Material Science and Engineering, 300 (2001), 125-134.

25. T. M. Smith et al., "Phase Transformation Strengthening of High Temperature Superalloys," Pending Submission (2016).

26. V. A. Vorontsov et al., "High-resolution electron microscopy of dislocation ribbons in a CMSX-4 superalloy single crystal," Acta Materialia, 60 (2012), 4866-4878.

27. C. M. F. Rae and L. Zhang, "Primary creep in single crystal superalloys: some comments on effects of composition and microstructure," Material Science and Technology, 25 (2009), 228-235.

28. A. Ma, D. Dye, and R. C. Reed, "A model for the creep deformation behaviour of single-crystal superalloy CMSX-4," Acta Materialia, 56 (2008), 1657-1670.

29. R. R. Unocic et al., "Deformation Mechanisms in Ni-Base Disk Superalloys at Higher Temperatures," Superalloys 2008 (Eleventh Int. Symp.) (2008), 377-385.

30. H. Roth, C. Davis, and R. Thomson, "Modeling solid solution strengthening in nickel alloys," Metallurgy. Materials., 28 (1997), 1329-1335.

31. R. Unocic, "On the Creep Deformation Mechanisms of an Advanced Disk Nickel-Base Superalloy," (Ph.D Thesis, The Ohio State University, 2008) 